\title{
The Influence of Gate Scaling to Electrical Characteristics on n-MOS FinFET
}

\author{
Nuttapong Patchrasardtra , Weera Pengchan \\ Department of Electronics Engineering, Faculty of Engineering, King Mongkut's Institute of Technology Ladkrabang
}

\begin{abstract}
This paper investigates effects from gate scaling in Tri-gate FinFET structure by simulation method, to avoid problems and improve a structure to be good prototype. The experiments used GTS framework for simulation. Start from 20 $\mathrm{nm}$ device, then scaling to $22 \mathrm{~nm} 28 \mathrm{~nm}$ and $32 \mathrm{~nm}$. Therefrom Minimos-NT function has used for biasing to giving two electrical characteristics as the drain current saturation and the threshold voltage. From these consequences can offer the subthreshold swing and the drain-induced barrier lowering by calculation. The results found that threshold voltage inversely proportional to saturated drain current, the subthreshold swing and the drain-induced barrier lowering. The short channel effect has affected to $20 \mathrm{~nm}$ model by highest DIBL. Therefore should be adjust the gate length and the oxide thickness properly to improve this effect.
\end{abstract}

\section{Introduction}

The introduced of Tri-gate transistor technology by Intel corporation in $2^{\text {nd }}$ quarterly of 2011 . Intel brought the new technology to giving good performance for them devices. 3-D transistor as known as FinFET. This is a choice to make new computer architecture. In $2011 \mathrm{M}$. Zakir Hossain and his researcher team shown electrical characteristics of SOI tri-gate FinFET. The result of this paper explain about basic characteristics such as the current and voltage characteristics (I-V), the threshold voltage, the electron mobility and field mobility [1]. In 2012 they proposed about the drain-induced barrier lowering (DIBL) and short channel effect (SCE). Influence of DIBL and SCE is coming together. DIBL make a drain to gate electron barrier lower. In a same way I-V and threshold voltage will strange from theory, and downward a subthreshold characteristics. It make device cannot change to cut-off stage at low voltage [2]. This concept make author interested in effects from scaling a device to solve problems for improved a structure of FinFET prototype and fabrication in the future.

\section{Theory}

\subsection{Saturation Drain Current $\left(I_{d s, S a t}\right)$}

While the drain current has no longer affected by the drain voltage, and where the FinFET acts more like a current source, has called the current saturation. This current value can be solve by [3]:

$$
I_{d s}=\mu C_{o x} \frac{W_{g}}{L_{g}}\left(\frac{v_{g s}-v_{t h}}{2 m}\right)^{2}
$$

$\mathrm{W}_{\mathrm{g}}$, gate width $(\mathrm{nm}) \mathrm{L}_{\mathrm{g}}$, gate length $(\mathrm{nm}) \mathrm{C}_{\mathrm{ox}}$, Gate Oxide capacitance $(\mathrm{F} / \mathrm{nm})$

\subsection{Threshold Voltage $\left(\mathrm{V}_{\mathrm{th}}\right)$}

The minimum gate-to-source voltage differential that was needs to create a conducting path between the source and drain. This is the most significant characteristic that provide the value of power that used to switch the FinFET from off stage to on stage and show the switching performance of devices. The threshold voltage can giving from [4]:

$$
V_{t h}=\Phi+\frac{k T}{q} \ln \left(\frac{2 C_{o x} k T}{q^{w} n_{i} t_{s i}}\right)+\frac{h^{2} \pi^{2}}{2 m W_{s i}^{2}}
$$

$\mathrm{n}_{\mathrm{i}}$, Electron and Hole Concentration without Doping $\left(\mathrm{cm}^{-3}\right)$ $\mathrm{t}_{\mathrm{si}}$, Silicon Thickness (nm) h, Channel Height (nm) $\mathrm{w}_{\mathrm{si}}$, Silicon width $(\mathrm{nm}) \eta$, Carrier Concentration $\left(\mathrm{cm}^{-3}\right)$.

\subsection{Subthreshold Swing (SS)}

The subthreshold swing is logarithm of drain current relationship of drain current and gate voltage that 
determine the voltage per decade. The solution to find a subthreshold swing, can calculate with equation (3) [5] by methods in Figure 1.

$$
S S=v_{g s 2}-v_{g s 1}
$$

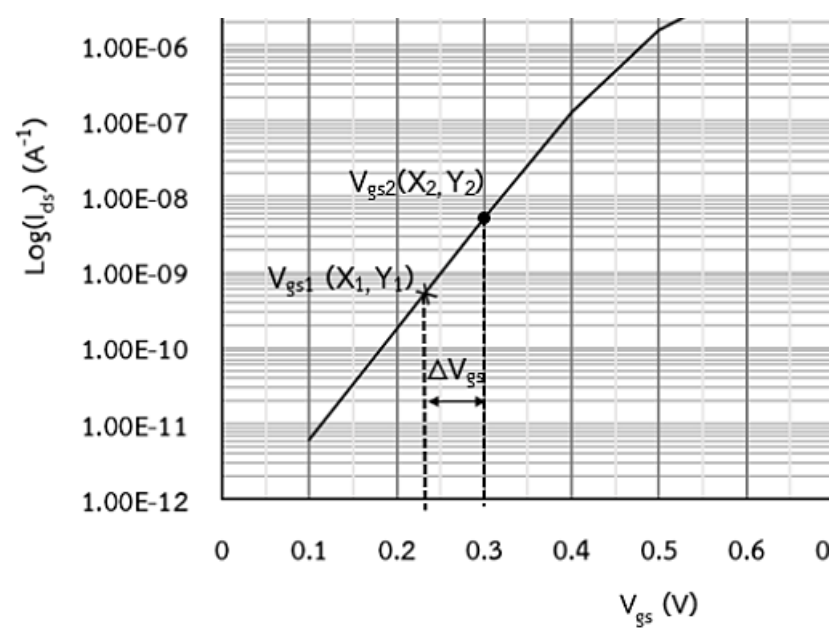

Figure 1. Relationship between the drain current and the gatesource voltage

\subsection{Drain-Induced barrier lowering (DIBL)}

The reduction of threshold voltage at higher drain voltages by the lowering of potential barrier on drain that affect to gate and Solve by [6]

$$
D I B L=\frac{\mathcal{V}_{t h}-\mathcal{V}_{t h}^{\text {Low }}}{\mathcal{V}_{d d}-\boldsymbol{V}_{d d}^{\text {Low }}}
$$

\section{Experimental}

This paper separated to two experiments part consist of the FinFET structure and the relationship between the electrical characteristics with the gate length. The first part, create a device structure on GTS framework [7] from $20 \mathrm{~nm}$ gate length and then increase $\mathrm{L}_{\mathrm{g}}$ to $22 \mathrm{~nm}, 28$ $\mathrm{nm}$, and $32 \mathrm{~nm} 10 \mathrm{~nm}$ silicon layer width and $25 \mathrm{~nm}$ silicon thickness with $1 \mathrm{~nm}$ gate oxide thickness on the top of $70 \mathrm{~nm}$ p-type substrate with buried oxide (BOX). In second part, Minimos-NT has use for biasing to obtain the electrical characteristics by determined the drain voltage at 1 volt and sweep the gate voltage from 0 to 1 volt ( 0.1 volt per steps) for harvest the threshold voltage. Collect the I-V characteristics by fixed the drain voltage at 1 volt and vary the gate voltage from 0 to 1 volt $(0.1$ volt per steps) and calculate the saturation regions of drain current. After collected $\mathrm{V}_{\text {th }}$ and $\mathrm{I}-\mathrm{V}$, calculate the subtheshold slop from $\log \left(\mathrm{I}_{\mathrm{ds}}\right) / \mathrm{V}_{\mathrm{gs}}$ and DIBL from $\mathrm{V}_{\text {th }}$ finally compare with gate length to giving the results of gate scaling effect.

\section{Result and Discussion}

\subsection{FinFET Structure}

From the first experiment part, created a FinFET structure that consist of the substrate, BOX, the silicon layer, channel, gate oxide and gate contact shown in Figure 2. (a) Side view of device with the gate length. (b) Cross section shown a fin width under oxide and contact, all of those are on buried oxide and the substrate. In this structure, determined the dopant concentration on channel at $1.5 \times 10^{15} \mathrm{~cm}^{-3}$ and $3 \times 10^{20} \mathrm{~cm}^{-3}$ on drain-source with poly-silicon gate contact therefore this part will provide four structures with difference of the gate length.

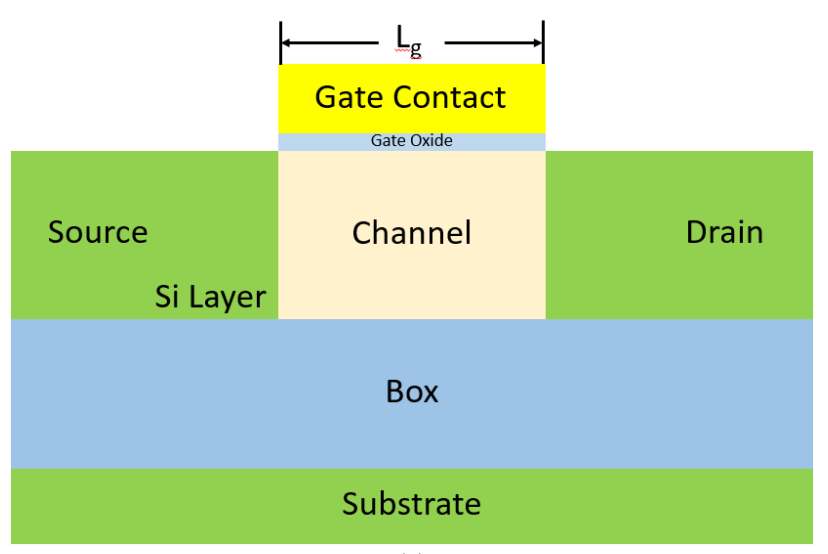

(a)

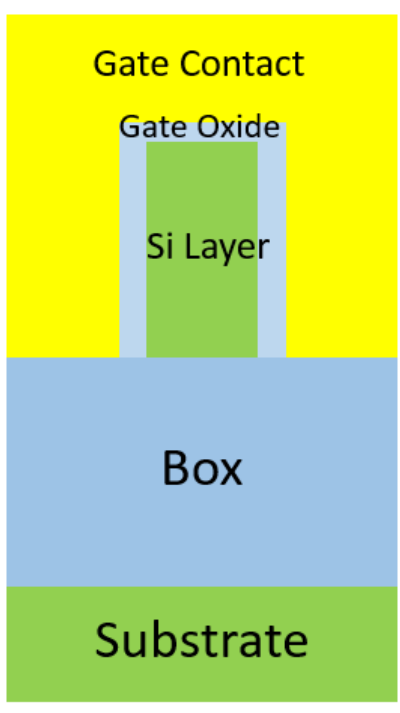

(b)

Figure 2. FinFET Structure (a) Side view (b) Cross Section

\subsection{Characteristics with gate length relationship}

The electrical characteristics of devices is the important point to determine a performance. From second part of experiment obtain the drain current saturation, the threshold voltage for calculate the subthreshold swing and the drain induced barrier lowering, to comparebetween characteristics and enlargement of gate length. 


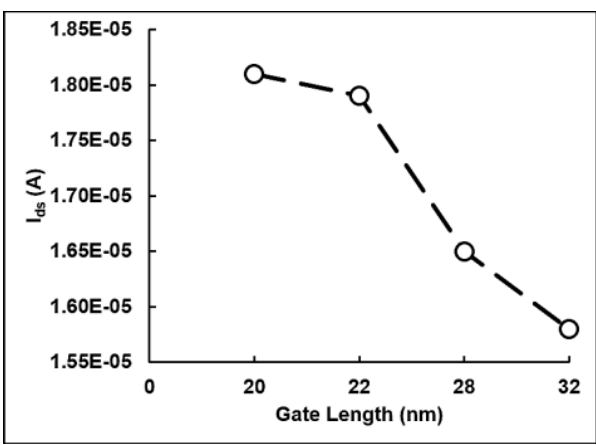

Figure 3. Drain current saturation with gate length

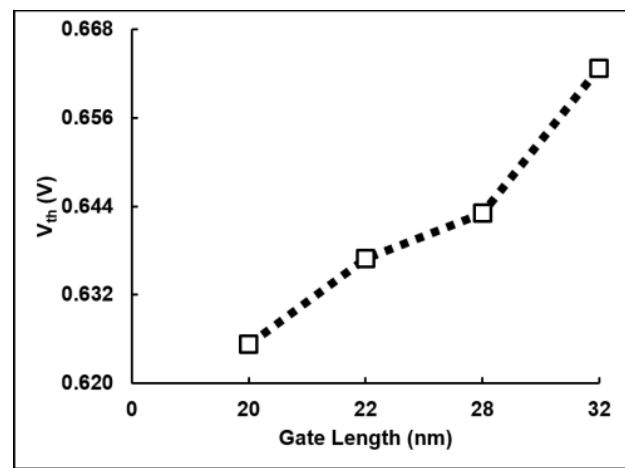

Figure 4. Threshold voltage with gate length

An enlargement of gate length determined a saturation current in Figure 3. The deportment of $\mathrm{I}_{\mathrm{ds}}$ was lowering with increase of $\mathrm{L}_{\mathrm{g}}$. Due to adding of electron concentration in channel that affect to them mobility. This effect bring $\mathrm{I}_{\mathrm{ds}}$ downward about $0.1 \mu \mathrm{A}$ per increasing of $\mathrm{L}_{\mathrm{g}}$. It was giving $\mathrm{I}_{\mathrm{ds}}$ on $20 \mathrm{~nm}$ ate length at $18.10 \mu \mathrm{A}, 22 \mathrm{~nm}$ at $17.90 \mu \mathrm{A}, 28 \mathrm{~nm}$ at $16.50 \mu \mathrm{A}$ and 32

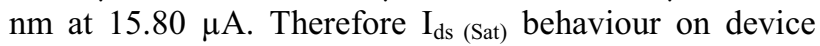
with $20 \mathrm{~nm} \mathrm{~L}_{\mathrm{g}}$ is better. In Figure 4, the threshold voltage has increased with $\mathrm{L}_{\mathrm{g}}$ but after biased $\mathrm{I}_{\mathrm{ds}} / \mathrm{V}_{\mathrm{gs}}$ giving amiss result of $20 \mathrm{~nm}$. It was coming with a little SCE because in this experiment vary only $\mathrm{L}_{\mathrm{g}}$. The threshold voltage of $20 \mathrm{~nm}$ gate length about 0.625 volt, $22 \mathrm{~nm}$ at 0.636 volt, $28 \mathrm{~nm}$ at 0.643 and $32 \mathrm{~nm}$ gate length has 0.662 volt thus SCE affect to this characteristics directly while gate length was increased. Normally, $V_{\text {th }}$ will increasing with $\mathrm{L}_{\mathrm{g}}$ since the gate length has increased and require more energy to turn device stage to turn-on.

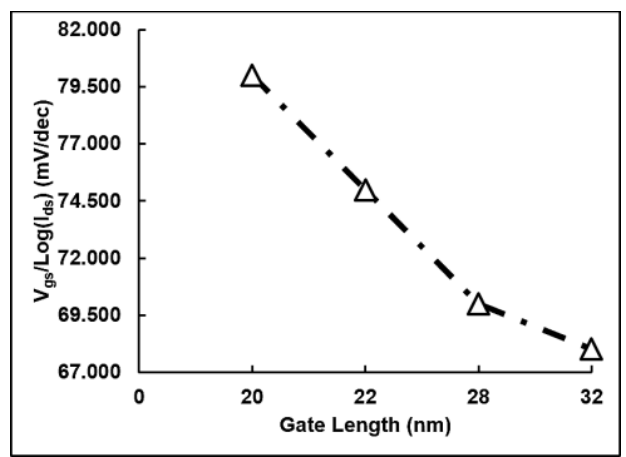

Figure 5. Subthreshold Swing with gate length

In Figure 5 shown the subthreshold swing has decreased by the gate length increasing, as more drain current passing in shorter $\mathrm{L}_{\mathrm{g}}$. From logarithm square root of drain current and gate-source voltage provide the SS on $20 \mathrm{~nm} \mathrm{~L}$ g $=80 \mathrm{mV} / \mathrm{dec}, 22 \mathrm{~nm}=75 \mathrm{mV} / \mathrm{dec}, 28 \mathrm{mV}=$ 70 and $32 \mathrm{~nm}$ gate length at $68 \mathrm{mV} / \mathrm{dec}$.

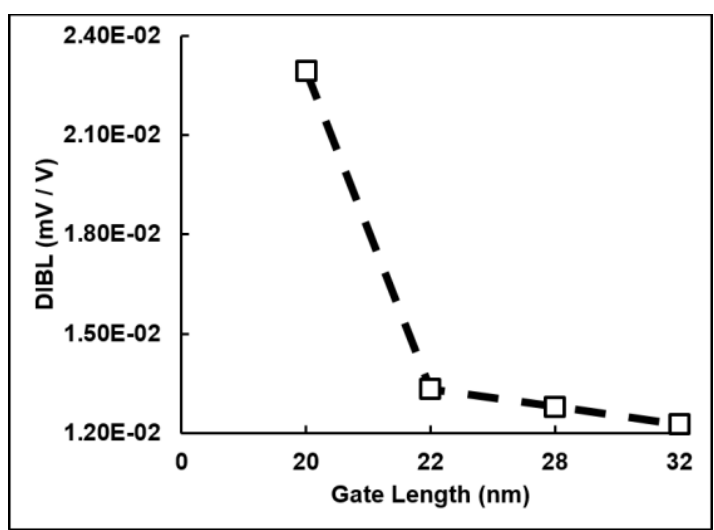

Figure 6. DIBL with gate length

The subthreshold swing is important attribute to obtain DIBL with gate length that shown in Figure 6. Influence of DIBL was directly to SS from lowering of barrier between channels and drain junction that affect to threshold voltage. DIBL on $20 \mathrm{~nm}$ gate length was about $23 \mathrm{mV} / \mathrm{V}, 22 \mathrm{~nm}$ at $13.30 \mathrm{mV} / \mathrm{V}, 28 \mathrm{~nm}$ at $12.80 \mathrm{mV} / \mathrm{V}$ and $32 \mathrm{~nm} \mathrm{~L}_{\mathrm{g}}$ approximate about $12.30 \mathrm{mV} / \mathrm{V}$. The DIBL giving to known a problem from SCE. If it was high, this device will be out of characteristics.

Table 1. Electrical characteristics of FinFET

\begin{tabular}{|c|c|c|c|c|}
\hline $\begin{array}{c}\text { Gate } \\
\text { Length } \\
(\mathbf{n m})\end{array}$ & $\begin{array}{c}\mathbf{I}_{\mathbf{d s}(\mathbf{s a t})} \\
(\boldsymbol{\mu A})\end{array}$ & $\begin{array}{c}\mathbf{V}_{\text {th }}(\mathbf{V}) \\
\left(\mathbf{V}_{\text {dd }}=\mathbf{1} \mathbf{V}\right)\end{array}$ & $\begin{array}{c}\mathbf{S S} \\
(\mathbf{m V} / \mathbf{d e c})\end{array}$ & $\begin{array}{c}\mathbf{D I B L} \\
(\mathbf{m V} / \mathbf{V})\end{array}$ \\
\hline $\mathbf{2 0}$ & 18.10 & 0.62 & 80.00 & 23.00 \\
\hline $\mathbf{2 2}$ & 17.90 & 0.63 & 75.00 & 13.30 \\
\hline $\mathbf{2 8}$ & 16.50 & 0.64 & 70.00 & 12.80 \\
\hline $\mathbf{3 2}$ & 15.80 & 0.66 & 68.00 & 12.30 \\
\hline
\end{tabular}

From Table 1, Increasing of gate length will bring the threshold voltage up but the drain current, the subthreshold swing and DIBL are inversely proportional. The highest DIBL of $20 \mathrm{~nm}$ giving a least $\mathrm{V}_{\text {th }}$ that affected by short channel effect not from decreasing of gate length.

\section{Summary}

Investigate into FinFET structure simulation is best way to understand operating of device. Many of problems has found from this experiment. In this article provides experiments to bring up effects from designed device structure, which are two effects as the short channel effect and the drain-induced barrier lowering. From the result found relationship between the drain current saturation, the threshold voltage, the subthreshold swing and the drain-induced barrier lowering. Increasing of gate length directly with $\mathrm{V}_{\text {th }}$ but reverse with $\mathrm{I}_{\mathrm{ds} \text { sat }}$, SS and 
DIBL. The short channel effect has found in $20 \mathrm{~nm}$ model therefore an adjustment of gate length will reach electrical characteristics suitability.

\section{Acknowledgement}

The authors would like to thank Electronic Research Center for many discussions and appreciate for software support from Global TCAD Solution.

\section{References}

1. M. Zakir Hossain and Md. Alamgir Hossain, Electrical Characteristics of Trigate Finfet Global, Journals of researches in Engineering Electrical and Electronics Engineering 11, 8-10 (2012)

2. M. Zakir Hossain and Quazi D. M. Khosru Threshold Voltage Roll-Off Due to Channel Length Reduction for a Nanoscale n-channel FinFET, IJETCAS 13-125, 152-156 (2012)
3. Viranjay M. Srivastava and Setu P. Singh, Analysis and Design of Tri-Gate MOSFET with High-k Dielectrics Gate I.J. Intelligent Systems and Applications 5, 16-22 (2012)

4. Nour El Islam and Boukortt Baghdad and Hadri Salvatore Patanè, Effects of High-k Dielectric Materials on Electrical Characteristics of DG nFinFETs, International Journal of Computer Applications, 139-10, 28-32 (2016)

5. Isabelle Ferain Cynthia A. Colinge and Jean-Pierre Colinge, Multigate transistors as the future of classical metal-oxide-semiconductor field-effect transistors, Nature 479, 310-316 (2011)

6. Yannis Tsividis, Operational Modeling of the MOS Transistor, 268 (1999)

7. Nuttapong Patcharasardtra and Weera Pengchan, Electrical Characteristics of Different Gate Geometry of FinFET, Key Engineering Materials 705, 174-178 (2016) 